\title{
Detection of Theileria luwenshuni in sheep from Great Britain
}

\author{
L. Paul Phipps ${ }^{1 *}$, Luis M. Hernández-Triana', Hooman Goharriz', David Welchman² and Nicholas Johnson
}

\begin{abstract}
Background: Theileria spp. are tick-borne protozoan parasites of the Phylum Apicomplexa, Order Piroplasmida that infect a wide range of wild and domestic animals. In Great Britain, Theileria spp. have been reported from livestock associated with transmission by the tick Haemaphysalis punctata. However, these reports have not been associated with disease. This study has investigated the cause of a disease outbreak accompanied by mortality in a flock of sheep grazing reclaimed marshland in north Kent.

Findings: A polymerase chain reaction-reverse line blot assay indicated the presence of Theileria spp. in blood samples from five animals. Subsequent testing with a pan-piroplasm PCR of a larger panel of blood samples detected a piroplasm amplicon in 19 of 21 sheep submitted from the affected flock. Automated sequencing confirmed that these amplicons shared 99-100\% identity with T. luwenshuni.
\end{abstract}

Conclusions: The clinical and PCR data suggest infection with T. luwenshuni was associated with disease and mortality in this flock.

Keywords: Theileria luwenshuni, Sheep, Great Britain, Haemaphysalis punctata

\section{Findings}

Theileria Species of Theileria are tick-borne, protozoan blood parasites of the Phylum Apicomplexa, Order Piroplasmida and along with the species of the closely related genus Babesia, may infect wild and domestic animals worldwide [1]. Theileria spp. infections are transmitted through the bite of infected ixodid ticks and are characterised by the formation of schizonts within the cytoplasm of host lymphocytes where asexual division takes place to form merozoites, which subsequently infect circulating erythrocytes to form piroplasms. Sexual reproduction occurs in the gut of the tick vector resulting in motile kinetes which invade the tick salivary gland where further division occurs in sporoblasts to form infective sporozoites [2]. Theileria parva and T. annulata, the causative agents of East Coast fever and Tropical theileriosis, respectively, are important pathogens of cattle in tropical and sub-tropical regions of the Old World [3]. Theileria equi causes equine piroplasmosis and has a worldwide distribution in tropical and sub-tropical regions [4]. In small

\footnotetext{
*Correspondence: Paul.Phipps@apha.gsi.gov.uk

${ }^{1}$ Wildlife Zoonoses and Vector-Borne Disease Research Group, Animal and Plant Health Agency, Woodham Lane, Addlestone, Surrey KT15 3NB, UK
} Full list of author information is available at the end of the article ruminants clinical disease has been historically associated with T. lestoquardi infection of sheep and described as malignant ovine theileriosis in the Mediterranean basin, north Africa and Asia [5]. Infection of goats with T. lestoquardi is reportedly benign. Recently, two new Theileria spp. causing clinical disease in sheep, T. uilenbergi and $T$. luwenshuni, have been described from northern China and associated with transmission by the hard tick Haemaphysalis qinghaiensis [6]. In a study to determine the prevalence and genetic diversity of piroplasms in northern Spain, three Theileria genotypes were identified in apparently healthy sheep, sharing 96.7-97.0 \% similarity between their $18 \mathrm{~S}$ rRNA gene sequences: $T$. ovis, $T$. sp. OT1 (99.6\% similarity with the recently described pathogenic piroplasm Theileria sp. China 1), and Theileria sp. OT3 [7]. In a more recent study, using a multiplex DNA bead-based suspension array (Luminex xMAP) to detect piroplasms in sheep from northern Spain, the authors detected five different piroplasms including Theileria sp. OT3 in $34.8 \%$ of the samples, T. ovis in $20.9 \%$, and at lower prevalences Babesia motasi (12.3 \%), T. luwenshuni/OT1 (10.5 \%) and Babesia ovis (6.3\%) [8]. During the two year period of this study, more than 10,000 questing ticks of all life-cycle 
stages were captured in the field and eight species of ticks identified including; Ixodes ricinus, $H$. punctata, Dermacentor reticulatus and $H$. inermis and sporadic captures of D. marginatus, I. frontalis, Rhipicephalus bursa and $H$. sulcata. The authors suggest a relationship between T. luwenshuni/OT1 and a Haemaphysalis sp. in the study area.

In Great Britain theileriosis has been reported from South and North Wales associated with transmission by $H$. punctata. Lewis and co-workers [9] isolated a piroplasm described as $T$. ovis when they inoculated a splenectomised sheep with a pooled blood sample taken from 50 healthy ewes grazing common land in coastal South Wales, where $H$. punctata was the only prevalent tick species. However, in North Wales, a morphologically similar piroplasm described as $T$. recondita was isolated by transmission via field-caught female $H$. punctata to splenectomised sheep [10]. The authors reported pyrexia with body temperatures rising to a maximum $41.5^{\circ} \mathrm{C}$ during peak phase of parasitaemia followed by recovery.

During April 2005 mortality associated with very heavy infestations by the tick $H$. punctata, was investigated in a group of 60 ewes and their lambs grazing north Kent marshland. More than 25 ewes died in the group which was part of a 900 ewe flock. However, it was observed that only the animals grazing one pasture where ticks were prevalent were affected. Post-mortem examination of an affected ewe revealed a very heavy burden of $H$. punctata, especially on the underside of the animal. The lips and tongue were swollen and oedematous, and there was also oedema of the conjunctivae and subcutaneous oedema around the eyes, and elsewhere over the face and throat. There were petechial haemorrhages over the oral mucosa and extensive subcutaneous congestion over the underside of the animal. The lungs were oedematous and mottled and excessive froth was present in the trachea and bronchi. The spleen was enlarged, the kidneys pale and the blood was watery.

Blood samples were taken by venepuncture into EDTA sample tubes from 2, 2-week old lambs and twelve random ewes in the same field. Giemsa staining of methanol fixed, thin blood smears was conducted using standard procedures [11]. This revealed the presence of anaplasmoid inclusions in the red cells of both of the lambs and ten of the ewes, accompanied by a moderate to severe macrocytic, hypochromic anaemia in the lambs and six of the ewes and a neutrophilia in the lambs and seven of the ewes. Further tests on blood samples from the sheep using PCR and reverse line blot (RLB) [12] at the University of Utrecht demonstrated the presence of a Babesia sp., a Theileria sp. and an Anaplasma / Ehrlichia sp. other than A. marginale, A. centrale, A. ovis and A. phagocytophilum. However, the University was unable to offer further specific molecular tests at the time.
During October 2012 a further 21 EDTA blood samples were taken from yearling sheep grazing the same pasture and subjected to a pan piroplasm PCR. Total DNA was extracted from $200 \mu \mathrm{l}$ whole blood using the DNAeasy Blood and Tissue Kit following the manufacturer's spincolumn protocol (QIAgen, Manchester, UK).

Pan Piroplasm PCR was conducted using the primers PIRO-A (5'-AATACCCAATCCTGACACAGGG-3') and PIRO-B (5'-TTAAATACGAATGCCCCCCAAC-3') [13] that gives a 423 base pair (bp) amplicon derived from the $18 \mathrm{~S}$ rRNA gene. Sample DNA was added to a mixture of primers and $\mathrm{SYBR}^{\circ}$ Green JumpStart ${ }^{\mathrm{Tm}}$ Taq ready mix (Sigma-Aldrich, St Louis, USA) in a total volume of $40 \mu \mathrm{l}$. Amplification was achieved with the following conditions: $94{ }^{\circ} \mathrm{C}$ for $10 \mathrm{~s}$ (s), followed by 45 cycles of $94{ }^{\circ} \mathrm{C}$ for $30 \mathrm{~s}, 58{ }^{\circ} \mathrm{C}$ for $30 \mathrm{~s}$ and $72{ }^{\circ} \mathrm{C}$ for $1 \mathrm{~min}$. Samples were separated on a $1 \%$ agarose gel containing SYBR safe (Sigma) and visualised by ultraviolet illumination. Samples were sequenced using the primers PIRO-A and PIRO-B with the ABI PRISM ${ }^{\circ}$ BigDye $^{\circ}$ Terminator v3.1 Cycle Sequencing Kit (Applied Biosystems, Life Technologies Ltd., Paisley UK) following the manufacturer's instructions. Neighbour-joining analysis was performed using MEGA v.5 software [14].

Nineteen samples produced a clear amplicon of a size corresponding with piroplasm controls (Fig. 1a). Each amplicon was sequenced using flanking primers. Sixteen of the 21 samples produced sequence suitable for further analysis. A representative sequence has been submitted to GenBank (Accession number KU234526). BLAST searches on each sequence indicated that they all shared $100 \%$ identity with T. luwenshuni (GenBank sequence: KP407010) and $99.8 \%$ identity with Theileria sp. OT1 (GenBank sequence: AY533143). Neighbour Joining (NJ) analysis of a subset of the Kent Theileria sequences compared with a range of ruminant Theileria spp. demonstrates the close association with $T$. luwenshuni with $100 \%$ bootstrap values (see Fig. 1b). The dataset was also analysed using the Maximum Likelihood method using bootstrap values of 1000 replications to assess phylogenetic relationships (tree not shown). We obtained a similar topology as the NJ tree with the group of T. luwenshuni supported with $100 \%$ bootstrap values.

Prior to the application of molecular phylogenetics to the delineation of blood parasites, Babesia motasi, $T$. recondita and Ehrlichia phagocytophila (now named Anaplasma phagocytophilum) were recognised tickborne haemoparasites of sheep in the United Kingdom (UK). Field surveys of the site detected the likely vector of T. luwenshuni, H. punctata, but failed to detect $T$. luwenshuni in a group of adults and nymphs. However, an amplicon of the correct size was detected in DNA extracted from one nymph and sequencing obtained 392 base pair sequence (GenBank Accession number 


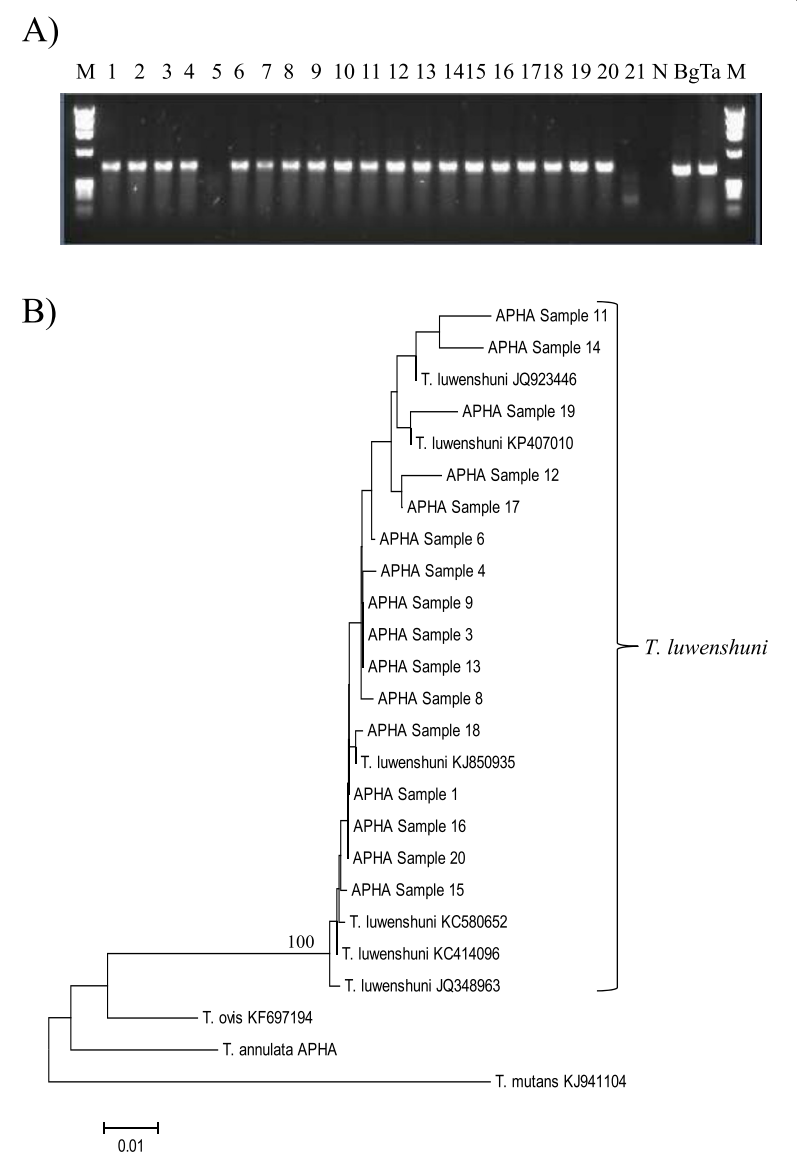

Fig. 1 a Gel analysis of pan-piro PCR on blood samples from Cooling Marsh sheep. Sheep samples are in tracks 1-21. M is DNA marker (fX154), N is a no-template negative control, Bg is B. gibsoni positive control and Ta is $T$. annulata positive control. b Neighbour Joining tree constructed from sequences (407 bp) of the $18 \mathrm{~S}$ rRNA gene including samples from infected ewes from Kent and representative Theileria sp. of ruminants. Only bootstrap values higher than $70 \%$ are shown in the tree below. Details of sequences included in the phylogeny are provided in Additional file 1: Table S1

KU234527). BLAST analysis, as described above, demonstrated that this amplicon shared $99 \%$ identity with B. motasi $18 \mathrm{~S}$ ribosomal subunit gene. This species is a relatively benign Babesia sp. previously described by Lewis and Herbert (1980) [15] from sheep in North Wales. In field conditions, $H$. punctata larvae feed on small mammals and birds and rarely feed on sheep [10]. It appears that transmission of T. luwenshuni is typical of the genus. The parasite is first picked up from infected carrier animals by the nymphal stage, which readily feeds on sheep, and passed on to the subsequent adult feeding stage i.e. transtadial transmission. In contrast, Babesia sp. transmission is transovarial. Female hard tick species acquire Babesia infection whilst feeding on infected carrier animals and pass the infection on to next generation larvae via the eggs. The subsequent life-cycle stages retain infection through feeding and metamorphosis and may transmit infection through to the first generation adult stage at least. This provides explanation for the detection of $B$. motasi in our field-caught $H$. punctata nymph. From the original RLB work carried out on this disease incident, it appears that an Ehrlichia / Anaplasma species may also subsequently be identified which has not been recorded in the UK until this time.

\section{Conclusions}

A pan-piroplasm PCR and subsequent sequencing of the amplicons suggests that the flock of sheep investigated in this study is infected with T. luwenshuni and that this could be the causative agent for disease and deaths observed on this farm in 2005. Clinical signs of acute babesiosis include fever, anaemia, icterus, haemogobinuria [16] whereas signs associated with acute or fatal theileriosis also include purulent nasal discharge, pneumonia, ataxia, dyspnoea in fatal T. orientalis infection in Asian water buffalos [17], fever, anaemia, serous nasal discharge, swelling of eyelids, ears and jowls, and pneumonia including frothy discharge from nostrils in the case of East Coast fever, caused by T. parva ([18]). The post mortem findings in the ewe and the moderate to severe anaemia in both the ewes and lambs were consistent with the reported effects of pathogenic Theileria spp. [19]. We conclude that T. luwenshuni has been indigenous to the UK and that previous investigations of cryptic ovine Theileria infection in Great Britain $[9,10]$ may be due to this species. Alternatively, T. luwenshuni could have been introduced from China where it is increasingly prevalent [20]. One potential source could have been the introduction of Asian deer species such as Sika (Cervus nippon), muntjac (Muntiacus reevesi) and Chinese water deer (Hydropotes inermis) over the past 150 years.

There has been no significant disease outbreak in the sheep on these marshes since 2005. The mortality in 2005 coincided with very heavy tick infestations, possibly triggered by the prevailing weather conditions. The ewes on this land were part of a closed flock and only rams were brought in for breeding purposes. This outbreak is consistent with an introduction of T. luwenshuni during 2004 via infected $H$. punctata nymphs on replacement rams followed by transmission by the subsequent adult population during the spring of 2005 causing clinical disease in a naïve flock of ewes and lambs. In following years the development of a stable endemicity [21] and subsequent low level of clinical disease may have been promoted through regular tick challenge of carrier ewes and protection of lambs by colostrum derived antibody, as suggested by the high infection rate of yearling sheep in this study. It is also feasible that heavy tick infestations may immunosuppress their hosts either via general 
debilitation and the effects of tick salivary proteins or via infection with known immunosuppressive tick-borne pathogens such as Anaplasma sp. thus promoting fulminating Theileria infection.

\section{Additional file}

Additional file 1: Table S1. Sample details of sequences used in the phylogeny of T. luwenshuni in the United Kingdom. (DOC 33 kb)

\section{Competing interests}

The authors declare that they have no competing interests.

\section{Authors' contributions}

LLP, NJ conceived and wrote the article. HG and LHT conducted molecular testing of samples and phylogenetic analysis. DW conducted field investigations and pathological assessment of infected sheep. All authors have read and approved the final version of the manuscript.

\section{Acknowledgements}

The authors would like to acknowledge Prof Frans Jongejan and Hein Sprong, University of Utrecht for testing samples by PCR-RLB, Andrew Sandilands and the clinical chemistry unit, Shrewsbury VI Centre for undertaking the haematology, John Kenward MRCVS for submitting the post mortem material and initial blood samples and the farmer, for allowing tick collection and blood sampling of his sheep. This study was funded by Defra, Scottish Government and Welsh Government under project SV3045.

\section{Author details}

${ }^{1}$ Wildlife Zoonoses and Vector-Borne Disease Research Group, Animal and Plant Health Agency, Woodham Lane, Addlestone, Surrey KT15 3NB, UK ${ }^{2}$ Surveillance Intelligence Unit, APHA Winchester, Itchen Abbas, Winchester SO21 1BX, UK

Received: 7 January 2016 Accepted: 1 April 2016

Published online: 13 April 2016

\section{References}

1. Yin H, Schnittger L, Luo J, Seitzer U, Ahmed JS. Ovine theileriosis in China: a new look at an old story. Parasitol Res. 2007;101:S191-5.

2. Mehlhorn, H., Schein, E. (1985) In: The Piroplasms: Life cycle and Sexual Stages. Advances in Parasitology APL, 23, 4th Edition. Cambridge, Massachusetts, USA: Academic Press, pp. $38-105$

3. Mans BJ, Pienaar R, Latif AA. A review of Theileria diagnostics and epidemiology. Int J Parasitol Parasites Wildlife. 2015;4:104-18.

4. Wise LN, Kappmeyer LS, Mealey RH, Knowles DP. Review of equine piroplasmosis. J Vet Intern Med. 2013;27:1334-46.

5. Levine ND. Apicomplexa: The Piroplasms. In: Veterinary Protozoology. Ames, IA: lowa State University Press; 1985. p. 291-328.

6. Yin H, Luo J, Guan G, Gao Y, Lu B, Zhang Q, Ma M, Lu W, Lu C, Yuan Z, Guo S, Wang B, Du H, Schnittger L, Ahmed J, Jongejan F. Transmission of an unidentified Theileria species to small ruminants by Haemaphysalis qinghaiensis ticks collected in the field. Parasitol Res. 2002:88:S25-7.

7. Nagore D, Garcia-Sanmartin J, Garcia-Pérez AL, Juste RA, Hurtado A. Identification, genetic diversity and prevalence of Theileria and Babesia species in a sheep population from Northern Spain. Int J Parasitol. 2004;34:1059-67.

8. Ros-García A, Barandika JF, García-Pérez AL, Juste RA, Hurtado A. Assessment of exposure to piroplasms in sheep grazing in communal mountain pastures by using a multiplex DNA bead-based suspension array. Parasites Vector. 2013;6:277-87.

9. Lewis D, Purnell RE, Bevan WJ. The piroplasm Theileria ovis detected in sheep in South Wales. Vet Rec. 1981:108:56-7.

10. Alani AJ, Herbert IV. Pathogenesis of infection with Theileria recondita (Wales) isolated from Haemaphysalis punctata from North Wales. Vet Parasitol. 1988;28:293-301.

11. Tamura K, Peterson D, Peterson N, Stecher G, Nei N, Kumar S. MEGA5: Molecular evolutionary genetics analysis using maximum likelihood, evolutionary distance and maximum parsimony methods. Mol Biol Evol. 2011;28:2731-39.

12. Gubbels JM, de Vos AP, van der Wiede M, Viseras J, Schouls LM, de Vries E, Jongejan F. Simultaneous detection of bovine Theileria and Babesia species by reverse line blot hybridization. J Clin Microbiol. 1999;37:1782-9.

13. Armstrong PM, Katavolos P, Caporale DA, Smith RP, Spielman A, Telford 3rd SR. Diversity of Babesia infecting deer ticks (Ixodes dammini). Am J Trop Med Hyg. 1998;58:739-42.

14. Tamura, K., Peterson, D., Peterson, N., Stoecher, G., Nei, M., Kumar, S. (2007) MEGA 5 (available at http://www.megasoftware.net)

15. Lewis D, Herbert IV. A large Babesia of sheep from North Wales. Vet Rec. 1980;107:352

16. Kakona I, Mehlhorn H. Babesia of domestic animals. In: Kreier JP, editor. Parasitic protozoa, part 2, vol. 7. San Diego, USA: Academic; 1994. p. 141-204.

17. Vinodkumar K, Shyma V, Justin DK, Ashok S, Anu JP, Mini K, Muhammedkutty V, Sasidharan S. Fatal Theileria orientalis N2 genotype infection among Asian water buffaloes (Bubalis bubalis) in a commercial dairy farm in Kerala, India. Parasitology 2016;143(1):69-74.

18. Mehlhorn H, Schein E, Ahmed JS. Theileria. In: Kreier JP, editor. Parasitic protozoa, part 2, vol. 7. San Diego, USA: Academic; 1994. p. 217-304.

19. Preston PM. Theileriosis. In: Service MW, editor. Encyclopaedia of arthropod transmitted infections in man and domestic animals. Wallingford, UK: CABI; 2001. p. 487-502.

20. Li Y, Zhang X, Lui Z, Chen Z, Yang J, He H, Guan G, Liu A, Ren Q, Niu Q, Liu J, Luo J, Yin H. An epidemiological survey of Theileria infections in small ruminants in central China. Vet Parasitol. 2014;200:198-200.

21. Norval RAl, Perry BD, Young AS. The epidemiology of theileriosis in Africa. ondon: London: Academic Press; 1992
Submit your next manuscript to BioMed Central and we will help you at every step:

- We accept pre-submission inquiries

- Our selector tool helps you to find the most relevant journal

- We provide round the clock customer support

- Convenient online submission

- Thorough peer review

- Inclusion in PubMed and all major indexing services

- Maximum visibility for your research

Submit your manuscript at www.biomedcentral.com/submit 